\title{
DIAGNOSES, OUTCOMES, AND CHRONICITY PREDICTORS OF PATIENTS WITH SECONDARY IMMUNE THROMBOCYTOPENIA: TEN-YEAR DATA FROM A HEMATOLOGY REFERRAL CENTER
}

José C. Jaime-Pérez*, Eugenia M. Ramos-Dávila, Patrizia Aguilar-Calderón, Raúl A. Jiménez-Castillo, AND DAVID GÓMEZ-ALMAGUER

Department of Hematology, Division of Internal Medicine, University Hospital "Dr. José Eleuterio González", School of Medicine, Universidad Autónoma de Nuevo León, Monterrey, NL, Mexico

\begin{abstract}
Background: Secondary immune thrombocytopenia (ITP) is a heterogeneous and unpredictable disease associated with various underlying conditions. Objective: The objective of the study was to investigate clinical evolution and chronicity predictors in secondary ITP. Methods: Patients treated at an academic medical center during 2008-2019 were stratified by age as children $<16$ years and adults $>16$ years. Responses to steroids, intravenous immunoglobulin G (IVIG), rituximab, and eltrombopag were classified as response (R) and complete response (CR). Risk factors for chronic ITP were determined by multiple regression with uni- and multi-variate analysis. Results: Eighty-three patients were included, 37 children and 46 adults. The most frequent associated conditions were infections 53\%, systemic lupus erythematosus (SLE) $24 \%$, thyroid disease $9.6 \%$, and Evans syndrome 3.6\%. Response to first-line treatment in the whole cohort was 94\%; CR 45.7\%; and R 50.6\%. Initial response to steroids alone was $91.3 \%(n=21 / 23)$, rituximab plus high-dose dexamethasone $(H D D) 93.3 \%(n=14 / 15)$; children receiving IVIG alone $100 \%(n=12 / 12)$; and eltrombopag in adults $100 \%(n=3 / 3)$. Relapse was documented in $19.4 \%$ of children and $34 \%$ of adults, at a median time of 15 and 2 months, respectively; $30.4 \%$ of adults ( $15.2 \%$ from the miscellaneous group, $10.9 \%$ SLE-associated, and $4.3 \%$ infection-associated) and $18.9 \%$ of children followed a chronic course; age $\geq 10$ years and platelets $\geq 20 \times 10^{9} / \mathrm{L}$ were risk factors for chronic ITP in children. Conclusion: Evolution was heterogeneous: a better and more sustained response was documented in the infections group compared to SLE or the miscellaneous group. (REV INVEST CLIN. 2021;73(1):31-8)
\end{abstract}

Key words: Autoimmune thrombocytopenia. Systemic lupus erythematosus. Steroids. Rituximab. Eltrombopag. Chronic.

*Corresponding author:

José C. Jaime-Pérez

E-mail: carjaime@hotmail.com
Received for publication: 12-05-2020

Approved for publication: 12-08-2020

DOI: $10.24875 /$ RIC. 20000214

0034-8376 / (c) 2020 Revista de Investigación Clínica. Published by Permanyer. This is an open access article under the CC BY-NC-ND license (http://creativecommons.org/licenses/by-nc-nd/4.0/). 


\section{INTRODUCTION}

Immune thrombocytopenia (ITP) is an autoimmune disease characterized by increased platelet consumption and reduced production with platelet counts $<100 \times 10^{9} / \mathrm{L}$ caused by both antibody and cell-mediated immune dysregulation ${ }^{1,2}$. ITP can be classified as primary, when no associated disease can be found, or secondary when ITP etiology is attributed to an underlying condition, including lymphoproliferative disorders, systemic lupus erythematosus (SLE), thyroid disease, antiphospholipid syndrome (APS), drug treatments, and infections such as human immunodeficiency virus (HIV), Helicobacter pylori infection, and hepatitis $\mathrm{C}$ or $\mathrm{B}$ virus $(\mathrm{HCV}, \mathrm{HBV})^{3}$. Although primary and secondary ITP share the generation of antiplatelet antibodies, they differ in specific aspects of pathobiology, natural history, and responsiveness to therapy ${ }^{4}$.

Secondary ITP diagnosis can be challenging due to the need to exclude the extensive differential diagnosis of thrombocytopenia and the lack of a specific diagnostic test. Recommendations for the initial diagnostic approach include patient history, physical examination, complete blood count, and peripheral blood smear examination. In addition, most authors routinely test adults for HCV, HBV, HIV, and, in appropriate geographical areas, $H$. pylori infection ${ }^{5}$. Autoimmune markers such as antinuclear antibody, rheumatoid factor, anticardiolipin antibodies, antithyroid peroxidase antibodies, and lupus anticoagulant have a higher prevalence in patients with ITP with no clinical evidence of these disorders; hence, they should not be routinely tested in the absence of diseasespecific symptoms, although their presence can be the harbinger of autoimmune disease ${ }^{6}$. In addition, antiplatelet antibodies are only detected in $50-60 \%$ of patients and bone marrow examination is not diagnostic; thus, they are not recommended as an initial approach $^{5-7}$.

Patients with primary or secondary ITP are often treated similarly. However, the current paradigm for secondary ITP may require treatment of the underlying disorder before treating thrombocytopenia ${ }^{3,5}$. Management might be difficult since corticosteroids and splenectomy are less effective than in primary ITP ${ }^{3}$. Establishing the diagnosis of secondary ITP requires precise targeting of the underlying disease, generally requiring an extensive clinical and laboratory workup.

We aimed to determine the incidence of secondary ITP over a decade at a referral hematology center, describe the underlying diseases, treatment modalities, long-term clinical outcomes, and chronic course predictors.

\section{METHODS}

This retrospective study included 258 consecutive patients diagnosed with ITP during the years 20082019. An underlying disease associated with secondary ITP was found in 83 (32\%) patients. All patients had complete information in their clinical records and electronic files and were treated at the Department of Hematology of the Dr. José Eleuterio González University Hospital and the School of Medicine of the Universidad Autónoma de Nuevo Leon in Monterrey, Mexico. The study was approved by the Ethics and Research Committees of the institution and was in full compliance with the principles of the Declaration of Helsinki as revised in 2013.

Patients were divided into children $<16$ years and adults $\geq 16$ years. The diagnosis was made according to clinical signs of bleeding and a platelet count $<100 \times 10^{9} / \mathrm{L}$.

Classification of disease stages was made following standard terminology, including newly diagnosed ITP when time between clinical symptoms and diagnosis was < 3 months; persistent ITP from 3 to 12 months since diagnosis; and chronic ITP when the disease lasted $\geq 12$ months. Diagnosis of severe ITP required the presence of clinically important bleeding manifestations ${ }^{5}$.

Initial treatment was selected by the treating physician according to the patient's clinical history, comorbidities, and bleeding severity. Responses were classified as complete response (CR) when platelets were $\geq 100 \times 10^{9} / \mathrm{L}$ without bleeding manifestations and response $(R)$ if there was a platelet count $\geq 30 \times 10^{9} / \mathrm{L}$ or a 2-fold increment from diagnosis without bleeding. No response (NR) was established by a platelet count $<30 \times 10^{9} / \mathrm{L}, \mathrm{a}<2$-fold increase of the baseline platelet count or bleeding. Loss of response was defined 
as a platelet count $<100 \times 10^{9} / \mathrm{L}$ or less than a 2 -fold increase of the baseline platelet count ${ }^{8}$.

For the purposes of this analysis, children in whom a viral illness was documented during the 2 weeks preceding the beginning of ITP symptoms were classified as having secondary ITP4,9,10. Primary disorders in adults were divided into three groups: infections, SLE, and miscellaneous.

\section{Statistical analysis}

The statistical analysis was carried out with SPSS v.22 (IBM SPSS Statistics software, IBM Corp., Armonk, NY). Categorical variables are displayed as absolute numbers and percentages and comparisons were made with the Pearson $x^{2}$ test. Quantitative variables were analyzed with descriptive statistics, including median and ranges. The Mann-Whitney $U$ test was used for comparisons between quantitative variables. Risk factors for chronicity were studied by logistic regression analysis with a $95 \%$ confidence interval $(\mathrm{Cl}) . A \mathrm{p}<0.05$ was considered statistically significant.

\section{RESULTS}

There were 83 patients with secondary ITP, 37 (44.5\%) children, $46(55.5 \%)$ adults; there were $52(62.6 \%)$ women and 31 (37.4\%) men, with a female to male ratio of $1.67: 1$ and a median follow-up of 21 (1-157) months. Overall response (OR) to first-line treatment was documented in $78(93.97 \%)$ patients.

\section{Pediatric Patients}

Thirty-seven children younger than 16 years diagnosed with secondary ITP were included. There were 22 (59.5\%) girls and 15 (40.5\%) boys. Children had a median age of $3(1-16)$ years with a median WHO bleeding scale of 1 (0-3). The main clinical characteristics are included in Table 1. Associated diseases in this group were previous infection in $32(86.4 \%)$ patients; 25 were associated with respiratory tract infection, 5 with varicella-zoster virus and 2 with gastrointestinal infection; secondary ITP (SLE-associated) was confirmed in 4 (10.8\%) children, 3 girls and 1 boy; and 1 boy (2.7\%) had Evans syndrome.
Of the 37 children, 13 (35.1\%) were treated exclusively with steroids, 12 (32.4\%) with a combination of intravenous immunoglobulin G (IVIG) plus steroids, $8(21.6 \%)$ received IVIG alone, $2(5.4 \%)$ danazol plus steroids, and $2(5.4 \%)$ children were under clinical observation. The initial response included 18 (48.6\%) CR and 18 (48.6\%) R; 1 (2.7\%) was a non-responder diagnosed with secondary ITP (varicella-zoster virusassociated). The median time to maximum response was 7 (2-31) days. Responses to each treatment are summarized in Table 2. One patient presented neutropenia after treatment with steroids plus IVIG.

Relapse was documented in 7 (19.4\%) children at a median of 15 (1-55) months. Six patients (85.7\%) were treated again with steroids, and one (14.3\%) with IVIG plus steroids; all achieved response. At last follow-up, 5 (13.5\%) children had persistent disease and $7(18.9 \%)$ followed a chronic course; of this last group, 4 (57\%) were infection-associated and 3 (43\%) SLE-associated. The median follow-up for children was 12.0 (range: $1-76$ ) months.

Risk factors for chronicity were analyzed by logistic regression with a $95 \% \mathrm{Cl}$. Uni- and multi-variate analysis results are shown in Table S1; age $\geq 10$ years and a platelet count $>20 \times 10^{9} / \mathrm{L}$ at diagnosis were statistically significant.

\section{Adults}

Secondary ITP diagnosis was established in 46 adults, $30(65.2 \%)$ women and $16(34.8 \%)$ men for a female to male rate of 1.9:1. A median age of 37 (17-73) years with a median WHO bleeding scale of $2(0-3)$ was found, clinical characteristics at diagnosis are shown in Table 1.

The underlying diseases associated with secondary thrombocytopenia were SLE in 16 (34.8\%) patients, $11(68.8 \%)$ women and 5 (31.2\%) men; infection in 12 (26.1\%); thyroid disease in $8(17.3 \%), 6$ with Graves' disease and 2 hypothyroidism; 2 (4.3\%) APS; and 2 (4.3\%) Evans syndrome. The remaining 6 patients had diagnoses, including HCV, fibromyalgia, psoriatic arthritis, connective tissue disease, papillary thyroid cancer, and one was vaccination-associated. H. pylori testing was performed in 12 patients and 6 (50\%) were positive. 
Table 1. Clinical characteristics and laboratory features of 83 patients with secondary ITP treated at a University Hospital in Northeast Mexico

\begin{tabular}{|c|c|c|}
\hline Variable & Children & Adults \\
\hline Total & 37 & 46 \\
\hline \multicolumn{3}{|l|}{ Gender (\%) } \\
\hline Male & $15(40.5)$ & $16(34.8)$ \\
\hline Female & $22(59.5)$ & $30(65.2)$ \\
\hline Age, years, median (range) & $3(0-16)$ & $37(17-73)$ \\
\hline \multicolumn{3}{|l|}{ Clinical characteristics (\%) } \\
\hline Petechiae & $26(70.3)$ & $21(45.7)$ \\
\hline Ecchymosis & $25(67.6)$ & $21(45.7)$ \\
\hline Gingivorrhagia & $4(10.8)$ & $18(39.1)$ \\
\hline Epistaxis & $12(32.4)$ & $11(23.9)$ \\
\hline Hematuria & $3(8.1)$ & $3(6.5)$ \\
\hline Hematemesis & $1(2.7)$ & - \\
\hline Lower Gl bleeding & $1(2.7)$ & $3(6.5)$ \\
\hline Metrorrhagia & - & $6(13.3)$ \\
\hline Hemorrhagic bullae & $1(2.7)$ & $2(4.3)$ \\
\hline Subconjunctival hemorrhage & - & $2(4.3)$ \\
\hline \multicolumn{3}{|c|}{ Laboratory features, median (range) } \\
\hline Platelets $\times 10^{9} / \mathrm{L}$ & $8.14(0.257-98)$ & $13.1(0.421-81)$ \\
\hline Leukocytes $\times 10^{9} / \mathrm{L}$ & $5(4.69-16.1)$ & $8.34(2.98-25)$ \\
\hline Neutrophils $\times 10^{9} / \mathrm{L}$ & $3.11(0-27.6)$ & $5.84(1.12-62)$ \\
\hline Lymphocytes $\times 10^{9} / \mathrm{L}$ & $3.03(0-65.9)$ & $1.94(0.55-52.1)$ \\
\hline Chronic course (\%) & $7(18.9)$ & $14(30.4)$ \\
\hline
\end{tabular}

ITP: immune thrombocytopenia

Table 2. Initial response in 37 children with secondary ITP receiving treatment at a reference center in Mexico

\begin{tabular}{lccc}
\hline Type of treatment & $\begin{array}{c}\text { Complete response, } \\
\mathbf{n}(\%)\end{array}$ & $\begin{array}{c}\text { Response, } \\
\mathbf{n}(\%)\end{array}$ & $\begin{array}{c}\text { No-Response, } \\
\mathbf{n}(\%)\end{array}$ \\
\hline Steroids alone & $8(61.5)$ & $4(30.7)$ & $1(7.6)$ \\
IVIG alone & $3(37.5)$ & $5(62.5)$ & - \\
IVIG plus steroids & $6(50)$ & $6(50)$ & - \\
Danazol plus steroids & - & $2(100)$ & - \\
Clinical Observation & $1(50)$ & $1(50)$ & - \\
\hline
\end{tabular}

ITP: immune thrombocytopenia; IVIG: intravenous immunoglobulin G.

Of the 46 adults, 10 (21.7\%) were treated exclusively with steroids, 15 (32.6\%) received rituximab at low doses ( $100 \mathrm{mg} / \mathrm{m}^{2} /$ week/4 weeks) plus steroids, $8(17.4 \%)$ danazol plus steroids, 1 (2.2\%) eltrombopag plus steroids, 2 (4.3\%) eltrombopag plus steroids plus low doses of rituximab, 2 (4.3\%) IVIG plus steroids, $4(8.7 \%)$ rituximab plus danazol plus steroids, and 4 (8.7\%) were under clinical observation. Response was achieved in $95.65 \%$ of the patients. CR was documented in 20 (43.48\%) and R in 
Table 3. Initial response in 46 adults with secondary immune thrombocytopenia at Dr. José Eleuterio González University Hospital in Mexico

\begin{tabular}{lccc}
\hline Treatment & $\begin{array}{c}\text { Complete response, } \\
\mathbf{n}(\%)\end{array}$ & $\begin{array}{c}\text { Response, } \\
\mathbf{n}(\%)\end{array}$ & $\begin{array}{c}\text { No-response, } \\
\mathrm{n}(\%)\end{array}$ \\
\hline Steroids alone & $5(50)$ & $4(40)$ & $1(10)$ \\
Rituximab plus HDD & $5(33.3)$ & $9(60)$ & $1(6.6)$ \\
Danazol plus steroids & $2(25)$ & $6(75)$ & - \\
Eltrombopag plus HDD & $1(100)$ & $1(50)$ & - \\
Eltrombopag plus rituximab + HDD & $1(50)$ & $2(50)$ & - \\
Rituximab plus danazol + HDD & $2(50)$ & - & - \\
IVIG plus steroids & $2(100)$ & $2(50)$ & - \\
Clinical Observation & $2(50)$ & & - \\
\hline
\end{tabular}

HDD: High dose dexamethasone; IVIG: intravenous immunoglobulin G.

Figure 1. Median platelet count during the first 6 months of follow-up in 46 adults with secondary immune thrombocytopenia (ITP) associated with systemic lupus erythematosus (SLE), infection, or miscellaneous diseases. SLE-associated ITP had the worse course.

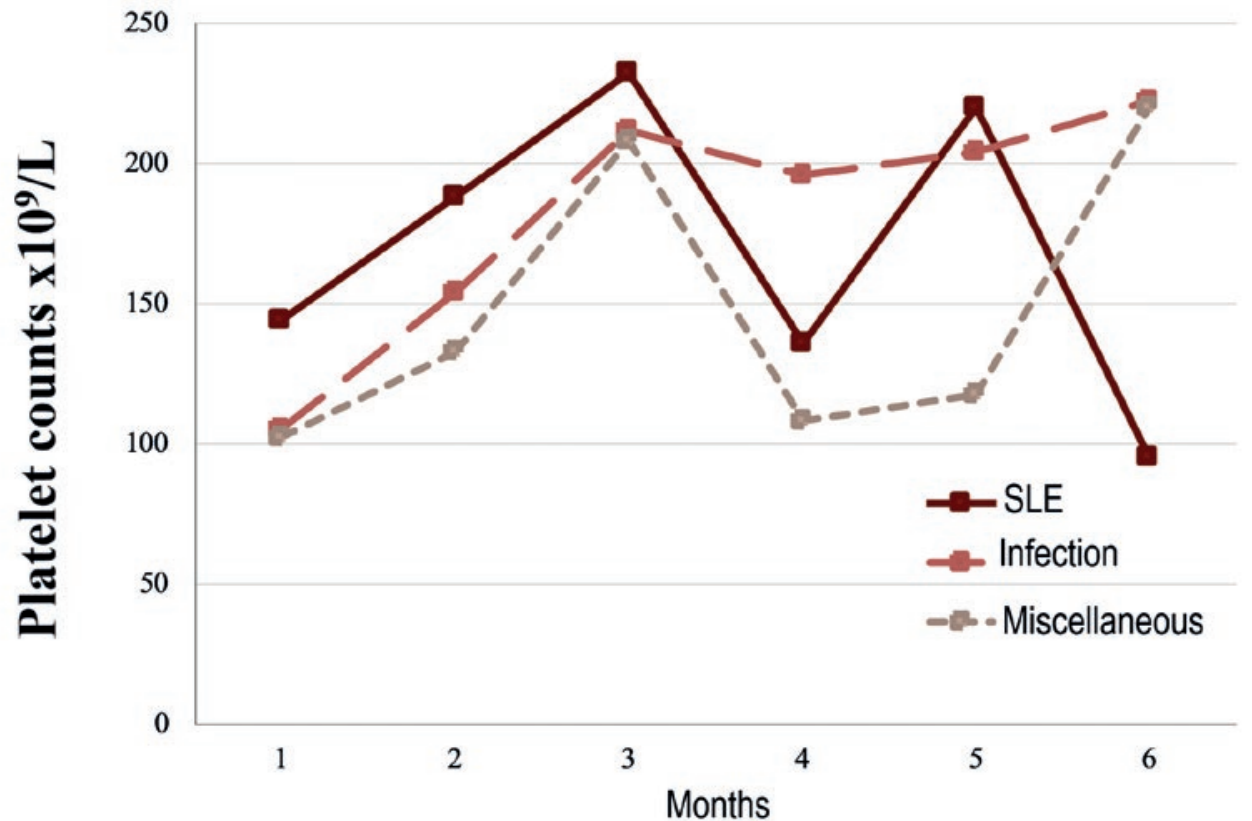

24 (52.17\%); there were $2(4.35 \%)$ NR diagnosed with secondary ITP (SLE-associated) and (thyroid disease-associated). The median time to maximum response was 6 (1-45) days. Responses to each treatment are summarized in Table 3 . Adverse effects of treatment were observed in 3 adults, including hyperglycemia, acne, and weight gain.

The cohort was further divided into 3 groups according to the associated disease; 16 (34.8\%) SLE, 12 (26\%) infection, and 18 (39.1\%) miscellaneous causes mentioned above. Secondary ITP (SLE-associated) patients and those in the miscellaneous group presented a drop in the platelet count at 4 months of follow-up with a median decrement of 96 and $100 \times$ $10^{9} / \mathrm{L}$, respectively. Nonetheless, the median platelet count remained higher than $100 \times 10^{9} / \mathrm{L}$. The platelet count course is shown in figure 1 .

Fifteen $(34.1 \%)$ of the 44 patients that reached a response relapsed at a median of $2(0.2-70)$ months; $6(40 \%)$ were treated again with steroids, 8 (53.3\%) 
with rituximab plus high-dose dexamethasone; and 1 (9.1\%) had a splenectomy. Fourteen (93.3\%) achieved a second response, except for 1 (6.7\%) patient with an underlying infectious disease receiving steroids only.

At last follow-up, 4 (8.69\%) adult patients had persistent disease, and 14 (30.43\%) progressed to a chronic course, 8 (57\%) women and 6 (43\%) men. In those who followed a chronic course, 5 (35.7\%) had SLE, 4 (28.5\%) thyroid disease, 2 (14.3\%) Evans syndrome, 2 (14.3\%) infection, and 1 (7.1\%) APS, displayed in figure $\mathrm{S} 1$. The median follow-up for adults was 26 (range: 1-157) months. The clinical course according to a secondary cause is shown in Table S2. No significant difference was found among the three groups.

By logistic regression analysis, no factor was found to be statistically significant for chronicity in adults with secondary ITP, Table S3.

\section{DISCUSSION}

ITP is a relatively common disease in the geographic region of the study, being the second and third most frequent hematological diagnosis in children and adults, respectively ${ }^{11}$. Secondary ITP prevalence varies greatly worldwide, with an estimated $20 \%$ of all ITP cases in the United States compared to $32 \%$ in the present report ${ }^{10}$. Studies dealing with demographic characteristics, clinical findings, treatment alternatives, and long-term outcomes in secondary ITP are scarce, with a predominance of information on its primary form. Findings in our study cohort contribute to define these features.

We gathered information on 83 patients of all ages over a decade. Secondary ITP was slightly more common in adults and females, as reported in previous studies $^{12}$. The median platelet count at diagnosis was 8.14 and $13.1 \times 10^{9} / \mathrm{L}$ in children and adults, respectively, considerably lower than $18.1 \times 10^{9} / \mathrm{L}$ and $25.4 \times 10^{9} / \mathrm{L}$ reported in primary ITP ${ }^{13}$. It is known that most children do not have serious bleeding problems despite low platelet counts, as confirmed in our study where children presented lower platelet counts and a median WHO bleeding scale of
1 compared to 2 in the adult group ${ }^{5}$. These findings support theories of a higher platelet threshold for bleeding in children, probably due to enhanced platelet function ${ }^{14}$.

The CR to first-line treatment was $93.97 \%$, higher than $70-90 \%$ reported in primary ITP5,15. The median time to response was 6-7 days, considerably faster than the 15 days reported in a study where only eltrombopag was used ${ }^{12}$. In the present study, eltrombopag was successfully administrated combined with steroids or with steroids plus low dose rituximab in three adult patients; all achieved an initial response. These treatment regimens have been previously studied as first-line treatment for primary ITP with encouraging results, but further evaluation is needed regarding its role in secondary ITP 16,17 .

Chronic ITP ensued in 30\% adults and $19 \%$ children, lower than $50-70 \%$ and $20-25 \%$ reported in primary ITP, respectively $7,18,19$. To the best of our knowledge, there are no previous studies regarding progression to chronic disease in secondary ITP; some authors suggest its course is expected to be more complicated than in the primary disease, our study documents the contrary ${ }^{3,4}$.

Risk factors for chronicity in children were age $>10$ years, as previously reported in primary ITP, and platelet counts $\geq 20 \times 10^{9} / \mathrm{L}$ at diagnosis ${ }^{19}$. Paradoxically, an increased platelet count at diagnosis as a risk factor for chronicity could be associated to an epitope spreading mechanism upregulated by the underlying disease. This could relate to the overall lower incidence of chronic ITP in our study with the lower platelet counts presented in our patients compared with primary ITP studies in other populations ${ }^{13,20}$.

Depending on the underlying disease, a great diversity of pathways lead to immune dysregulation. These pathways can be divided into central (bone marrow) defects, as seen in autoimmune diseases, or peripheral defects associated with chronic underlying conditions such as infections ${ }^{10}$. It is thought that peripheral defects have a better response rate to treatment and our study appears to support this concept (83.7\% in SLE vs. $100 \%$ in infections). However, this difference did not reach statistical significance. In addition, chronic ITP was more frequent in SLE-associated and thyroid disease-associated ITP than in the infections 
group, supporting the rationale of a more severe immune dysregulation associated with autoimmune diseases than a transitory peripheral defect in acute and chronic infections ${ }^{10}$.

ITP appears as a secondary complication in $10-40 \%$ of SLE patients, while $25 \%$ of secondary ITP cases are associated with SLE ${ }^{10,21,22}$. This is comparable to $24 \%$ observed in our study and expectedly lower than the estimated $61.9 \%$ when considering all forms of rheumatic diseases reported in Mexico ${ }^{23}$. Thyroid disease is associated with $5 \%$ of secondary ITP cases $^{10}$, whereas we found a prevalence of $17.4 \%$. Evans syndrome is an uncommon disease reported in $0.7-2 \%$ of secondary ITP cases; in our report, it accounted for $3.6 \%$, closer to the $4 \%$ found in a Pakistani popula$\operatorname{tion}^{10,22,24}$.

Infection-associated ITP was found in 53\% (44/83) of our entire cohort, similar to $52.4 \%$ estimated in an all-age electronic survey in Mexico ${ }^{23}$. Children were more prone to infection-associated ITP; 32 (86.4\%) referred to a previous acute infection and $75 \%$ of them achieved complete remission, comparable with the prevalence in children of $66 \%$ with a remission rate of $80 \%$ reported in other series ${ }^{4,10}$.

H. pylori infection prevalence varies worldwide, with ranges from $1 \%$ in the United States to $60 \%$ in Japan and Italy ${ }^{4,10}$. In this study, H. pylori test was performed when the primary disorder remained unclear; therefore, only 12 patients were tested and 50\% $(6 / 12)$ presented a positive result, similar to $60 \%$ $(14 / 23)$ found in a Mexican population ${ }^{25}$, and higher than $31 \%$ in an international study and $5 \%$ in the United States ${ }^{10,13}$. In addition, SLE patients have been reported to have a $39 \%$ prevalence of $H$. pylori infection $^{26}$. In this study, only three SLE patients were tested with two positive results $(66.6 \%)$. The benefits of routinely testing for $H$. pylori and its eradication need to be further studied.

Only one case (2\%) was vaccination-associated, it presented on a 32-year-old woman with a recent hepatitis $B$ and influenza vaccination in the previous 5 days. Vaccination has been reported to account for $5 \%$ of secondary ITP cases ${ }^{4,10}$. There are few case reports of influenza vaccination associated with ITP10,27,28, and it is unknown whether simultaneous vaccination leads to an increased risk for ITP.
Adult patients from the SLE and miscellaneous groups presented a drop in median platelet counts at four months of follow-up, as opposed to the infections group, which had a more consistent platelet response. Interestingly, this equals the median time to relapse of 4 months seen in the SLE-associated group of the present study. Another report found a less fluctuating median platelet count through time; however, the platelet range in that study was notoriously wider around the $4^{\text {th }}$ month of follow-up ${ }^{12}$. Therefore, stricter control during the first 4 months of treatment could be warranted.

Secondary ITP associated with autoimmune diseases was notoriously more prone to follow a chronic course than when associated with infections, probably due to the ongoing autoimmune process; this supports the theory of different pathways triggering a similar clinical presentation. Further studies are needed to deepen the understanding of these mechanisms, which could lead to improved targeted treatment according to the primary disease.

\section{ACKNOWLEDGEMENTS}

We would like to thank Sergio Lozano-Rodriguez, MD (Faculty of Medicine, Universidad Autónoma de Nuevo León, NL, Mexico), for his critical review of the manuscript.

\section{SUPPLEMENTARY DATA}

Supplementary data are available at Revista de Investigación Clínica online (www.clinicalandtranslationalinvestigation.com). These data are provided by the corresponding author and published online for the benefit of the reader. The contents of supplementary data are the sole responsibility of the authors.

\section{REFERENCES}

1. Iraqi M, Perdomo J, Yan F, Choi PY, Chong BH. Immune thrombocytopenia: antiplatelet autoantibodies inhibit proplatelet formation by megakaryocytes and impair platelet production in vitro. Haematologica. 2015;100:623-32.

2. Mckenzie CG, Guo L, Freedman J, Semple JW. Cellular immune dysfunction in immune thrombocytopenia (ITP). Br J Haematol. 2013;163:10-23.

3. Bussel JB. Therapeutic approaches to secondary immune thrombocytopenic purpura. Semin Hematol. 2009;46:S44-58. 
4. Cines DB, Liebman $H$, Stasi R. Pathobiology of secondary immune thrombocytopenia. Semin Hematol. 2009;46:S2-14.

5. Provan D, Arnold DM, Bussel JB, Chong BH, Cooper N, Gernsheimer $\mathrm{T}$, et al. Updated international consensus report on the investigation and management of primary immune thrombocytopenia. Blood Adv. 2019;3:3780-817.

6. Hollenhorst MA, Al-Samkari H, Kuter DJ. Markers of autoimmunity in immune thrombocytopenia: prevalence and prognostic significance. Blood Adv. 2019;3:3515-21.

7. Cooper N, Ghanima W. Immune thrombocytopenia. N Engl J Med. 2019:381:945-55.

8. Rodeghiero F, Stasi R, Gernsheimer T, Michel M, Provan D, Arnold $\mathrm{DM}$, et al. Standardization of terminology, definitions and outcome criteria in immune thrombocytopenic purpura of adults and children: report from an international working group. Blood. 2009;113:2386-93.

9. Blanchette V, Bolton-Maggs P. Childhood immune thrombocytopenic purpura: diagnosis and management. Hematol Oncol Clin North Am. 2010;24:249-73.

10. Cines DB, Bussel JB, Liebman HA, Prak ET. The ITP syndrome: pathogenic and clinical diversity. Blood. 2009:113:6511-21.

11. Jaime-Pérez JC, Treviño-Reyna G, Aguilar-Calderón P, CantúRodríguez OG, Marfil-Rivera LJ, Gómez-Almaguer D. Contributions of a regional approach to document hematologic disease in Mexico: a 10-year experience in an open population. Hematology. 2018;23:803-9.

12. González-López TJ, Alvarez-Román MT, Pascual C, SánchezGonzález B, Fernández-Fuentes F, Pérez-Rus G, et al. Use of eltrombopag for secondary immune thrombocytopenia in clinical practice. Br J Haematol. 2017;178:959-70.

13. Kühne T, Berchtold W, Michaels LA, Wu R, Donato H, Espina B, et al. Newly diagnosed immune thrombocytopenia in children and adults: a comparative prospective observational registry of the intercontinental cooperative immune thrombocytopenia study group. Haematologica. 2011;96:1831-7.

14. Nobuko N, Hirokazu K, Akuta K, Hayashi S, Kato H, Kanakura Y, et al. Reevaluation of platelet function in chronic immune thrombocytopenia: impacts of platelet size, platelet-associated anti- $\alpha \mathrm{llb} \beta 3$ antibodies and thrombopoietin receptor agonists. $\mathrm{Br}$ J Haematol. 2020;189:760-71.

15. Frederiksen $H$, Christiansen CF, Norgaard M. Risk and prognosis of adult primary immune thrombocytopenia. Expert Rev Hematol. 2012;5:219-28.

16. Gómez-Almaguer D. Eltrombopag-based combination treatment for immune thrombocytopenia. Ther Adv Hematol. 2018; 9:309-17.

17. Gómez-Almaguer D, Colunga-Pedraza PR, Gómez-De León A, Gutiérrez-Aguirre $\mathrm{CH}$, Cantú-Rodríguez OG, Jaime-Pérez JC. El- trombopag, low-dose rituximab, and dexamethasone combination as frontline treatment of newly diagnosed immune thrombocytopaenia. Br J Haematol. 2019;184:288-90.

18. Grimaldi-Bensouda L, Nordon C, Michel M, Viallard JF, Adoue D, Magy-Bertrand $\mathrm{N}$, et al. Immune thrombocytopenia in adults: a prospective cohort study of clinical features and predictors of outcome. Haematologica. 2016;101:1039-45.

19. Heitink-Pollé KM, Nijsten J, Boonacker CW, de Haas M, Bruin MC. Clinical and laboratory predictors of chronic immune thrombocytopenia in children: a systematic review and meta-analysis. Blood. 2014;124:3295-307.

20. Al-Samkari H, Rosovsky RP, Leaf RS, Smith DB, Goodarzi K, Fogerty $A E$, et al. A modern reassessment of glycoprotein-specific direct platelet autoantibody testing in immune thrombocytopenia. Blood Adv. 2020;4:9-18.

21. Jung JH, Soh MS, Ahn YH, Um YJ, Jung JY, Suh CH, et al. Thrombocytopenia in systemic lupus erythematosus: clinical manifestations, treatment, and prognosis in 230 patients. Medicine (Baltimore). 2016;95:e2818.

22. Sultan S, Ahmed SI, Murad S, Irfan SM. Primary versus secondary immune thrombocytopenia in adults; a comparative analysis of clinical and laboratory attributes in newly diagnosed patients in Southern Pakistan. Med J Malaysia. 2016;71:269-74.

23. Meillón-García LA, García-Chávez J, Gómez-Almaguer D, Gutiérrez-Espíndola GR, Martínez-Murillo C. Trombocitopenia inmune primaria (TIP) del adulto en México: características nacionales y su relación con la literatura internacional. Gac Med Mex. 2014:150:279-88.

24. Jaime-Pérez JC, Aguilar-Calderón PE, Salazar-Cavazos L, Gómez-Almaguer D. Evans syndrome: clinical perspectives, biological insights and treatment modalities. J Blood Med. 2018; 9:171-84.

25. Estrada-Gómez R, Parra-Ortega I, Martínez-Barreda C, RuizArgüelles GJ. Helicobacter pylori infection and thrombocytopenia: a single-institution experience in Mexico. Rev Investig Clin. 2007;59:112-5

26. Mendoza-Pinto C, García-Carrasco M, Méndez-Martínez S, Mogollán-Delfín T, Munguía-Realpozo P, Herrera-Robles E, et al. Helicobacter pylori infection and gastroduodenal lesions in patients with systemic lupus erythematosus. Clin Rheumatol. 2020;39:463-9.

27. Mitta A, Curtis BR, Reese JA, George JN. Drug-induced thrombocytopenia: 2019 update of clinical and laboratory data. Am J Hematol. 2019;94:E76-8.

28. Perricone C, Ceccarelli F, Nesher G, Borella E, Odeh Q, Conti F, et al. Immune thrombocytopenic purpura (ITP) associated with vaccinations: a review of reported cases. Immunol Res. 2014; 60:226-35 